\title{
On the minimizing movement with the 1-Wasserstein distance
}

\author{
Martial AgUeH ${ }^{* \dagger} \quad$ Guillaume CARLIER ${ }^{\ddagger} \quad$ Noureddine IGBIDA $\S$
}

August 30, 2017

\begin{abstract}
We consider a class of doubly nonlinear constrained evolution equations which may be viewed as a nonlinear extension of the growing sandpile model of [15]. We prove existence of weak solutions for quite irregular sources by a semi-implicit scheme in the spirit of the seminal works of [13] and [14] but with the 1-Wasserstein distance instead of the quadratic one. We also prove an $L^{1}$-contraction result when the source is $L^{1}$ and deduce uniqueness and stability in this case.
\end{abstract}

Keywords: 1-Wasserstein distance, minimizing movement, $L^{1}$-contraction, growing sandpiles.

MS Classification: 35K55, 35D30, 49N15.

\section{Introduction}

Given a convex nonlinearity $F$, and $\Omega$, an open bounded subset of $\mathbb{R}^{d}$, we are interested in (a suitable weak notion of solution for) the following evolution system:

$$
\partial_{t} u-\operatorname{div}\left(a \nabla F^{\prime}(u)\right)=f,(t, x) \in(0, T) \times \Omega,\left.u\right|_{t=0}=u_{0},
$$

*Department of Mathematics and Statistics, University of Victoria, P.O. Box. 3060 STN CSC, Victoria B.C., V8W 3R4, Canada. E-mail: agueh@math.uvic.ca

${ }^{\dagger}$ This work was completed after Martial passed away. We wish to dedicate this article to his memory.

${ }^{\ddagger}$ Ceremade, UMR CNRS 7534, Université Paris Dauphine, Pl. de Lattre de Tassigny, 75775, Paris Cedex 16, France, and MOKAPLAN, INRIA-Paris, E-mail: carlier@ceremade.dauphine.fr

§Institut de recherche XLIM-DMI, UMR-CNRS 7252, Faculté des Sciences et Techniques, Université de Limoges 123, Avenue Albert Thomas 87060 Limoges, France. Email: noureddine.igbida@unilim.fr 
supplemented with the zero flux condition i.e. the requirement that $a \nabla F^{\prime}(u)$ is tangential to $\partial \Omega$ and constrained by

$$
a \geq 0,\left|\nabla F^{\prime}(u)\right| \leq 1, a\left(1-\left|\nabla F^{\prime}(u)\right|\right)=0 .
$$

At least formally, (1.1)-(1.2) can be viewed as the limit as $q \rightarrow \infty$ of the doubly nonlinear evolution equation:

$$
\partial_{t} u-\Delta_{q}\left(F^{\prime}(u)\right)=f,\left.\quad u\right|_{t=0}=u_{0}
$$

where $\Delta_{q}$ is the $q$-Laplace operator, $\Delta_{q} v=\operatorname{div}\left(|\nabla v|^{q-2} \nabla v\right)$. In the linear case where $F^{\prime}(u)=u$, this equation arises as a model for growing sandpiles introduced by Prigozhin [15] and very much studied since, see in particular [2], [11], [5], [6], [10], [9], [12] and the references therein.

We shall address existence of weak solutions to (1.1)-(1.2) by a simple constructive Euler scheme reminiscent of the seminal works of Jordan-Kinderlehrer and Otto [13] and Kinderlehrer and Walkington [14] but with the 1-Wasserstein distance and not the more traditional quadratic one. Thanks to this point of view, we will obtain weak solutions for irregular sources $f$, namely $f \in L^{1}\left((0, T),\left(C^{0, \alpha_{0}}(\bar{\Omega})\right)^{\prime}\right)$. If the source is in fact $L^{1}$ in $t$ and $x$, then the flow of (1.1)-(1.2) defines a contraction in $L^{1}$ which implies uniqueness, stability as well as full convergence of the Euler scheme.

The paper is organized as follows. Section 2 is devoted to some preliminaries, the definition of weak solutions and a summary of our main results. Existence is established via a variational scheme à la Jordan-Kinderlehrer and Otto [13] and Kinderlehrer and Walkington [14] in section 3. In section 4 , an $L^{1}$-contraction result that implies uniqueness and stability for an $L^{1}$ source $f$ is proved. Finally, section 5 is devoted to some variants and concluding remarks.

\section{The PDE and its weak formulation}

\subsection{Preliminaries}

It is well-known that the constraints (1.2) are related to the 1-Wasserstein distance and the notion of Kantorovich potential. In the following, we assume that $\Omega$ is an open bounded convex subset of $\mathbb{R}^{d}$ and $T>0$. We denote by $\operatorname{Lip}_{1}$ the set of 1 -Lipschitz functions on $\Omega$, and by Lip $:=W^{1, \infty}(\Omega)$ the set of Lipschitz functions on $\Omega$. Given $g \in \operatorname{Lip}^{\prime}$ such that $g$ is balanced i.e.

$$
\langle g\rangle:=\langle g, 1\rangle=0,
$$

we denote by $W_{1}$ the dual semi norm of $g$ :

$$
W_{1}(g):=\sup _{\theta \in \operatorname{Lip}_{1}}\langle g, \theta\rangle .
$$


When $g$ is signed measure of the form $g=g^{+}-g^{-}$, with $g^{ \pm}$probability measures on $\bar{\Omega}$, $W_{1}(g)$ is the 1-Wasserstein distance between $g^{+}$and $g^{-}$(see [17]). Define

$$
\operatorname{Lip}_{0}^{\prime}:=\left\{g \in \operatorname{Lip}^{\prime}:\langle g\rangle=0\right\},
$$

a function $\theta \in \operatorname{Lip}_{1}$ for which $W_{1}(g)=\langle g, \theta\rangle$ is called a Kantorovich potential of $g$ and we denote by $\mathrm{K}(g)$ the set of Kantorovich potentials of $g$ i.e.:

$$
\mathrm{K}(g):=\left\{\theta \in \operatorname{Lip}_{1}:\langle g, \theta\rangle \geq\langle g, \psi\rangle, \forall \psi \in \operatorname{Lip}_{1}\right\} .
$$

For an arbitrary $g \in \operatorname{Lip}_{0}^{\prime}$, it may be the case that $\mathrm{K}(g)$ is empty. However, $\mathrm{K}(g) \neq \emptyset$ as soon as $g \in \operatorname{Lip}_{0}^{\prime} \cap X^{\prime}$ where $X$ is a space of functions such that the embedding from Lip to $X$ is compact (for instance $X=C^{0}, C^{0, \alpha}$ with $\alpha \in[0,1), \ldots$ ).

Using the Fenchel-Rockafellar duality theorem gives the following dual formula for $W_{1}(g)$ :

$$
W_{1}(g)=\inf _{\sigma \in\left(L^{\infty}\left(\Omega, \mathbb{R}^{d}\right)\right)^{\prime}}\left\{\|\sigma\|_{\left(L^{\infty}\right)^{\prime}}:-\operatorname{div}(\sigma)=g\right\}
$$

where the equilibrium condition $-\operatorname{div}(\sigma)=g$ has to be understood in the weak sense i.e.

$$
\langle\sigma, \nabla \theta\rangle_{L^{\infty^{\prime}, L^{\infty}}}=\langle g, \theta\rangle_{\mathrm{Lip}^{\prime}, \mathrm{Lip}}, \forall \theta \in W^{1, \infty}(\Omega) .
$$

For $g \in \operatorname{Lip}_{0}^{\prime}$, it also follows from the Fenchel-Rockafellar duality theorem that (2.1) admits solutions (in $\left(L^{\infty}\right)^{\prime}$ and not in $L^{1}$ in general), such solutions are called optimal flows. A Kantorovich potential $\theta \in \mathrm{K}(g)$ is related to an optimal flow $\sigma$ in (2.1) by the extremality relation

$$
\langle\sigma, \nabla \theta\rangle_{\left(L^{\infty}\right)^{\prime}, L^{\infty}}=\|\sigma\|_{\left(L^{\infty}\right)^{\prime}} \text { with }\|\nabla \theta\|_{L^{\infty}} \leq 1
$$

which, very informally, means that $\sigma$ is concentrated on the set where $|\nabla \theta|$ equals 1 and is collinear to $\nabla \theta$. If, by chance, $\sigma$ is $L^{1}$, the previous relation expresses the fact that $\sigma=a \nabla \theta$ with $a \geq 0$ as well as the complementary slackness condition $a(1-|\nabla \theta|)=0$, note also that $\sigma$ is in some weak sense tangential to $\partial \Omega$ because of (2.2). For an arbitrary

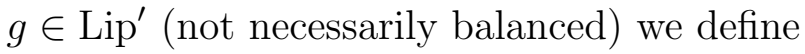

$$
\widetilde{W}_{1}(g):=W_{1}(g-\langle g\rangle)+|\langle g\rangle|
$$

and observe that $\widetilde{W}_{1}$ is equivalent to the usual norm of Lip'.

With the previous considerations in mind, it is natural to interpret the PDE (1.1) coupled with (1.2) as the inclusion

$$
-F^{\prime}(u) \in \mathrm{K}\left(\partial_{t} u-f\right),
$$


whose implicit in time discretization, given a time-step $\tau>0$, reads as

$$
-F^{\prime}\left(u_{k+1}\right) \in \mathrm{K}\left(u_{k+1}-u_{k}-\int_{k \tau}^{(k+1) \tau} f\right) .
$$

As we shall see later, these conditions appear as the Euler-Lagrange equations for the following Euler implicit scheme à la Jordan-Kinderlehrer-Otto ${ }^{1}$ (henceforth JKO) [13] but using $W_{1}$ instead of the more familiar 2-Wasserstein distance. Given a time step $\tau>0$, let us construct inductively a sequence $u_{k}^{\tau}$ by setting $u_{0}^{\tau}=u_{0}$ and

$$
u_{k+1}^{\tau} \in \operatorname{argmin}_{u}\left\{W_{1}\left(u-\left(u_{k}^{\tau}+\tau f_{k}^{\tau}\right)\right)+\int_{\Omega} F(u) \mathrm{d} x:\left\langle u-u_{k}^{\tau}-\tau f_{k}^{\tau}\right\rangle=0\right\}
$$

where (extending $f$ by 0 outside $[0, T]$ if necessary)

$$
f_{k}^{\tau}:=\frac{1}{\tau} \int_{k \tau}^{(k+1) \tau} f(s, .) \mathrm{d} s .
$$

From now on, in addition to the assumption that $\Omega$ is convex and bounded, we suppose that there exists $\alpha_{0} \in[0,1)$ such that ${ }^{2}$

$$
f \in L^{1}\left((0, T),\left(C^{0, \alpha_{0}}(\bar{\Omega})\right)^{\prime}\right)
$$

which in particular implies that

$$
\int_{0}^{T}|\langle f(t, .)\rangle| \mathrm{d} t+\int_{0}^{T} W_{1}(f(t, .)-\langle f(t, .)\rangle) \mathrm{d} t<+\infty .
$$

and to make things as elementary as possible we take a power nonlinearity for $F$ :

$$
F(u)=\frac{1}{m}|u|^{m}, \text { with } m>1
$$

and

$$
u_{0} \in L^{m}(\Omega) .
$$

It then follows directly from the fact that $W_{1}$ is lsc for the weak $L^{m}$ topology as well as the strict convexity of $F$ (and the convexity of $W_{1}$ ) that the sequence $u_{k}^{\tau}$ of the $W_{1}$-JKO

\footnotetext{
${ }^{1}$ the idea to incorporate the source in an explicit way in the scheme was actually introduced by Kinderlehrer and Walkington [14].

${ }^{2}$ note that this allows a rough dependence in $x$, not even a measure for instance $f(t,):.=\sum_{n}\left(\delta_{x_{n}(t)}-\right.$ $\left.\delta_{y_{n}(t)}\right)$ with $\int_{0}^{T} \sum_{n}\left|x_{n}(t)-y_{n}(t)\right|^{\alpha_{0}} \mathrm{~d} t<+\infty$.
} 
scheme (2.5) is uniquely well-defined. We define then two curves corresponding to linear and piecewise constant interpolation:

$$
u^{\tau}(t):=u_{k}^{\tau}+\frac{t-k \tau}{\tau}\left(u_{k+1}^{\tau}-u_{k}^{\tau}\right), \widetilde{u}^{\tau}(t):=u_{k+1}^{\tau}, t \in(k \tau,(k+1) \tau],
$$

for $k=0, \cdots,\left[\tau^{-1} T\right]$. We also define the piecewise constant approximation of the source $f:$

$$
f^{\tau}(t):=f_{k}^{\tau}, t \in[k \tau,(k+1) \tau), k=0, \cdots,\left[\tau^{-1} T\right] .
$$

Note that by construction

$$
\left\langle u_{k+1}^{\tau}\right\rangle=\left\langle u_{k+1}^{\tau}\right\rangle+\int_{k \tau}^{(k+1) \tau}\langle f(t, .)\rangle \mathrm{d} t
$$

so that with (2.8) and (2.10), we have

$$
\left\langle\widetilde{u}^{\tau}(t, .)\right\rangle,\left\langle u^{\tau}(t, .)\right\rangle \in L^{\infty}((0, T))
$$

\subsection{Weak solutions}

The notion of weak solution of (1.1)-(1.2) we consider heavily relies on (2.3) and the following (slightly formal) observations. Recall that (2.3) means that $F^{\prime}(u) \in \operatorname{Lip}_{1}$ and for every $\xi \in \operatorname{Lip}_{1}$, one has

$$
\left\langle\partial_{t} u(t), F^{\prime}(u(t))-\xi\right\rangle \leq\left\langle f(t), F^{\prime}(u(t))-\xi\right\rangle .
$$

Note that giving a pointwise in time sense to this condition would require that $\partial_{t} u \in$ $L^{1}\left((0, T)\right.$, Lip $\left.^{\prime}\right)$, which will not be guaranteed by the rather weak assumption $(2.7)$.

Defining for every $k>0$, the truncation map $T_{k}: \mathbb{R} \rightarrow \mathbb{R}$ by

$$
T_{k}(u):=\left\{\begin{array}{l}
k \text { if } u \geq k \\
u \text { if } u \in[-k, k] \\
-k \text { if } u \leq-k .
\end{array} .\right.
$$

and observing that for any $\theta \in \operatorname{Lip}_{1}, \xi:=F^{\prime}(u)-T_{k}\left(F^{\prime}(u)-\theta\right)$ belongs to $\operatorname{Lip}_{1}$ too, we get

$$
\begin{array}{r}
\left\langle\partial_{t} u, T_{k}\left(F^{\prime}(u)-\theta\right)\right\rangle=\frac{d}{d t} \int_{\Omega} \int_{0}^{u(t)} T_{k}\left(F^{\prime}(s)-\theta\right) \mathrm{d} s \mathrm{~d} x \\
\leq\left\langle f, T_{k}\left(F^{\prime}(u)-\theta\right)\right\rangle .
\end{array}
$$

These considerations lead to the following definition of weak solutions: 
Definition 2.1. A weak solution of (1.1)-(1.2) is an $u \in L^{\infty}\left((0, T), C^{0}(\Omega)\right)$ such that $F^{\prime}(u) \in \operatorname{Lip}_{1}$ for a.e. $t \in[0, T]$, and for every $\theta \in \operatorname{Lip}_{1}$ and every $k>0$,

$$
\frac{d}{d t} \int_{\Omega} \int_{0}^{u(t)} T_{k}\left(F^{\prime}(s)-\theta\right) d s d x \leq\left\langle f, T_{k}\left(F^{\prime}(u)-\theta\right)\right\rangle
$$

in the sense of distributions.

In other words, $u \in L^{\infty}\left((0, T), C^{0}(\Omega)\right)$ such that $F^{\prime}(u) \in \operatorname{Lip}_{1}$ for a.e. $t \in[0, T]$ is a weak solution of (1.1)-(1.2) if for every $\theta \in \operatorname{Lip}_{1}$ and every $\phi \in C_{c}^{1}\left([0, T), \mathbb{R}_{+}\right)$one has

$$
\begin{array}{r}
-\int_{0}^{T} \int_{\Omega} \dot{\phi}(t) \int_{0}^{u(t)} T_{k}\left(F^{\prime}(s)-\theta\right) \mathrm{d} s \mathrm{~d} x \mathrm{~d} t \leq \phi(0) \int_{\Omega} \int_{0}^{u_{0}} T_{k}\left(F^{\prime}(s)-\theta\right) \mathrm{d} s \mathrm{~d} x \\
+\int_{0}^{T} \phi(t)\left\langle f(t, .), T_{k}\left(F^{\prime}(u(t, .))-\theta\right)\right\rangle \mathrm{d} t .
\end{array}
$$

Note that if $u$ is a weak solution (letting $k \rightarrow \infty$ ), we have for every $\theta \in \operatorname{Lip}_{1}$

$$
\frac{d}{d t} \int_{\Omega} F(u(t, x)) \mathrm{d} x \leq \frac{d}{d t}\langle\theta, u\rangle+\left\langle f, F^{\prime}(u)-\theta\right\rangle
$$

in the sense of distributions, which implies in particular that $t \mapsto \int_{\Omega} F(u(t, x)) \mathrm{d} x$ as well as $t \mapsto\langle u(t,),. \theta\rangle$ (with $\theta \in \operatorname{Lip}$ ) are BV functions (but not necessarily absolutely continuous).

Remark 2.2. One can see here that the notion of solution we are using in Definition 2.1 is weaker than the standard one which consists in requiring that $u \in L^{\infty}\left((0, T), C^{0}(\Omega)\right)$, with $\partial_{t} u \in L^{1}\left(0, T ; \operatorname{Lip}^{\prime}\right)$ and $F^{\prime}(u(t)) \in \operatorname{Lip}_{1}$ for a.e. $t \in[0, T], u(0)=u_{0}$ and

$$
-F^{\prime}(u(t)) \in \mathrm{K}\left(\partial_{t} u(t)-f(t)\right), \quad \text { for a.e. } t \in(0, T) .
$$

Indeed, by using the same arguments of Lemma 4 of [7], one can prove that if $u$ is such a solution it is also a solution in the sense of Definition 2.1. Indeed, if $\partial_{t} u \in L^{1}\left(0, T ; \mathrm{Lip}^{\prime}\right)$ one can prove rigorously that (2.15) yields

$$
\begin{gathered}
\int_{0}^{T}\left\langle\partial_{t} u(t), T_{k}\left(F^{\prime}(u(t))-\theta\right)\right\rangle \phi(t) \mathrm{d} t=-\int_{0}^{T} \int_{\Omega} \dot{\phi}(t) \int_{0}^{u(t)} T_{k}\left(F^{\prime}(s)-\theta\right) \mathrm{d} s \mathrm{~d} x \mathrm{~d} t \\
-\phi(0) \int_{\Omega} \int_{0}^{u_{0}} T_{k}\left(F^{\prime}(s)-\theta\right) \mathrm{d} s \mathrm{~d} x,
\end{gathered}
$$

for any $\theta \in \operatorname{Lip}_{1}$ and $\phi \in C_{c}^{1}([0, T), \mathbb{R})$. 


\subsection{Main results}

Our main results concerning the existence and uniqueness of weak solutions can be summarized as follows. First, existence will be obtained (proof will be detailed in section 3) by convergence of the JKO-scheme:

Theorem 2.3. Assume (2.7)-(2.9)-(2.10) and define $u^{\tau}$ by (2.5)-(2.11), then there exists $u \in L^{\infty}\left((0, T), C^{0}(\Omega)\right)$ such that $F^{\prime}(u) \in \operatorname{Lip}_{1}$ for a.e. $t \in[0, T]$, and a vanishing family of stepsizes $\tau_{n} \rightarrow 0$ such that $u^{\tau_{n}}$ converges strongly to $u$ in $L^{p}\left((0, T), C^{0}(\bar{\Omega})\right)$ for every $p \in[1, \infty)$ and $u$ is weak solution of (1.1)-(1.2).

Uniqueness will be guaranteed by the following $L^{1}$-contraction result (see section 4 ) which requires an $L^{1}$ assumption on the source:

Theorem 2.4. Assume $f \in L^{1}\left((0, T), L^{1}(\Omega)\right)$, (2.9), $u_{0} \in L^{m}(\Omega), v_{0} \in L^{m}(\Omega)$ and let $u$ and $v$ be weak solutions of of (1.1)-(1.2) associated respectively to the initial conditions $u_{0}$ and $v_{0}$ respectively, then

$$
\|u(t, .)-v(t, .)\|_{L^{1}(\Omega)} \leq\left\|u_{0}-v_{0}\right\|_{L^{1}(\Omega)}, \text { a.e. } t \in[0, T] .
$$

In particular (1.1)-(1.2) has a unique weak solution.

Combining theorems 2.3 and 2.4 and the a priori estimates for the JKO scheme of section 3, we deduce:

Corollary 2.5. Assume that $f \in L^{1}\left((0, T), L^{1}(\Omega)\right)$, (2.9) and (2.10) then the whole family $u^{\tau}$ defined by (2.5)-(2.11) converges in $L^{p}\left((0, T), C^{0}(\bar{\Omega})\right)$ for every $p \in[1, \infty)$ to the unique solution of (1.1)-(1.2).

\section{Convergence of the JKO-like $W_{1}$-scheme}

\subsection{Euler-Lagrange equation for the discrete scheme}

The fact that the Euler-Lagrange equation of the variational problem in (2.5) is very much linked to an implicit time discretization of (2.3) follows from:

Lemma 3.1. Let $\alpha_{0} \in[0,1)$ and $v \in\left(C^{0, \alpha_{0}}(\bar{\Omega})\right)^{\prime}$, if $u \in L^{m}$ solves

$$
\inf \left\{W_{1}(u-v)+\int_{\Omega} F(u): u \in L^{m},\langle u-v\rangle=0\right\}
$$

then

$$
-F^{\prime}(u) \in \mathrm{K}(u-v) .
$$


Proof. We proceed by duality. Consider the convex minimization problem

$$
\inf _{z \in \operatorname{Lip}_{1}}\left\{\int_{\Omega} F^{*}(-z)+\langle v, z\rangle\right\}
$$

It is easy to see that it admits a (unique by strict convexity of $F^{*}$ ) solution, indeed if $z_{n}$ is a minimizing sequence, it possesses a subsequence that converges strongly in $C^{0, \alpha_{0}}(\bar{\Omega})$ (and thus also in $L^{m^{\prime}}$ with $m^{\prime}=\frac{m}{m-1}$ the conjugate exponent of $m$ ) to some $z$ which obviously solves (3.3) since $v \in\left(C^{0, \alpha_{0}}(\bar{\Omega})\right)^{\prime}$. By Fenchel-Rockafellar Theorem, we also deduce that (3.3) (written as a convex minimization problem on $L^{m^{\prime}}$ observing that $\operatorname{Lip}_{1}$ is closed in $L^{m^{\prime}}$ ) is dual to (3.1). Moreover, the solution $z \in \operatorname{Lip}_{1}$ of (3.3) is related to the solution $u$ of (3.1) by the extremality relation

$$
\begin{aligned}
0 & =\int_{\Omega} F(u)+W_{1}(u-v)+\int_{\Omega} F^{*}(-z)+\langle v, z\rangle \\
& =\int_{\Omega} F(u)+\int_{\Omega} F^{*}(-z)+\langle u, z\rangle+W_{1}(u-v)-\langle u-v, z\rangle
\end{aligned}
$$

since both terms are nonnegative this gives $z=-F^{\prime}(u)$ and $z \in \mathrm{K}(u-v)$.

Proposition 3.2. If for $i=1,2, v_{i} \in\left(C^{0, \alpha_{0}}(\bar{\Omega})\right)^{\prime}$ and $v_{1}-v_{2} \in L^{1}(\Omega)$, then the corresponding solutions $u_{i}$ to (3.1) satisfy

$$
\int_{\Omega}\left|u_{1}-u_{2}\right| \leq \int_{\Omega}\left|v_{1}-v_{2}\right|
$$

Proof. It follows from Lemma 3.1, that for $i=1,2$, we have

$$
\left\langle v_{i}-u_{i}, F^{\prime}\left(u_{i}\right)-\xi\right\rangle \geq 0, \quad \text { for any } \xi \in \operatorname{Lip}_{1},
$$

and since $F^{\prime}\left(u_{1}\right)-T_{k}\left(F^{\prime}\left(u_{1}\right)-F^{\prime}\left(u_{2}\right)\right) \in \operatorname{Lip}_{1}$, we have

$$
\left\langle v_{1}-u_{1}, T_{k}\left(F^{\prime}\left(u_{1}\right)-F^{\prime}\left(u_{2}\right)\right)\right\rangle \geq 0 .
$$

Similarly,

$$
\left\langle v_{2}-u_{2}, T_{k}\left(F^{\prime}\left(u_{2}\right)-F^{\prime}\left(u_{1}\right)\right)\right\rangle \geq 0 \text {. }
$$

hence

$$
\left\langle u_{1}-u_{2}, T_{k}\left(F^{\prime}\left(u_{2}\right)-F^{\prime}\left(u_{1}\right)\right)\right\rangle \leq\left\langle v_{1}-v_{2}, T_{k}\left(F^{\prime}\left(u_{2}\right)-F^{\prime}\left(u_{1}\right)\right)\right\rangle .
$$

Dividing both terms by $k$, using the fact that $v_{1}-v_{2} \in L^{1}$ and that $\frac{1}{k}\left|T_{k}\left(F^{\prime}\left(u_{1}\right)-F^{\prime}\left(u_{2}\right)\right)\right| \leq$ 1 , we get

$$
\frac{1}{k} \int_{\Omega}\left(u_{1}-u_{2}\right) T_{k}\left(F^{\prime}\left(u_{1}\right)-F^{\prime}\left(u_{2}\right)\right) \leq \int_{\Omega}\left|v_{1}-v_{2}\right|
$$


At last, letting $k \rightarrow 0$ and using Lebesgue's dominated convergence theorem and the fact that $F^{\prime}$ is increasing, we obtain (3.4).

As a consequence, we deduce that the discrete JKO scheme contracts the $L^{1}$ distance. Let us indeed consider the same $J K O$ construction (2.5) as before but for two different initial conditions $u_{0}$ and $v_{0}$, we denote by $u_{k}^{\tau}$ and $v_{k}^{\tau}$ the corresponding discrete in time sequences. We then have:

Corollary 3.3. The discrete JKO scheme given by (2.5) satisfies the discrete flow equation (2.4) and contracts the $L^{1}$ distance (whatever the time step $\tau>0$ is). In other words, the sequences $u_{k}^{\tau}$ and $v_{k}^{\tau}$ constructed by the scheme (2.5) corresponding to the initial conditions $u_{0}$ and $v_{0}$ satisfy

$$
\left\|u_{k+1}^{\tau}-v_{k+1}^{\tau}\right\|_{L^{1}} \leq\left\|u_{k}^{\tau}-v_{k}^{\tau}\right\|_{L^{1}}
$$

for any $k=0,1, \ldots\left[\tau^{-1} T\right]-1$.

Now, in order to pass to the limit in (2.4) for the discrete JKO scheme, as $\tau \rightarrow 0$, we give in this paragraph the main a priori estimates on $u^{\tau}$ and $\widetilde{u}^{\tau}$. Lemma 3.1 first gives the estimate

$$
\left\|\nabla\left[F^{\prime}\left(\widetilde{u}^{\tau}\right)\right]\right\|_{L^{\infty}((0, T) \times \Omega)} \leq 1 .
$$

Since $\left[F^{\prime}\right]^{-1}$ is $C^{0, \beta}$ for the exponent $\beta:=\min \left(1, \frac{1}{m-1}\right)$, thanks to $(2.13)$, we also have the Hölder bound:

$$
\left\|\widetilde{u}^{\tau}\right\|_{L^{\infty}\left((0, T), C^{0, \beta}(\Omega)\right)}+\left\|u^{\tau}\right\|_{L^{\infty}\left((0, T), C^{0, \beta}(\Omega)\right)} \leq C .
$$

Using $u_{k}^{\tau}+\tau\left\langle f_{k}^{\tau}\right\rangle$ as a competitor to $u_{k+1}^{\tau}$ in (2.5), we first have:

$$
\begin{aligned}
W_{1}\left(u_{k+1}^{\tau}-\left(u_{k}^{\tau}+\tau f_{k}^{\tau}\right)\right)+\int_{\Omega} F\left(u_{k+1}^{\tau}\right) & \leq \int_{k \tau}^{(k+1) \tau} W_{1}(f(t, .)-\langle f(t, .)\rangle) \mathrm{d} t \\
& +\int_{\Omega} F\left(u_{k}^{\tau}+\tau\left\langle f_{k}^{\tau}\right\rangle\right) .
\end{aligned}
$$

Thanks to (3.7) $u_{k}^{\tau}$ is bounded and thanks to $(2.8)$

$$
\tau\left|\left\langle f_{k}^{\tau}\right\rangle\right| \leq\|\langle f\rangle\|_{L^{1}(k \tau,(k+1) \tau)} \leq \omega(\tau) \text { with } \omega(\tau) \rightarrow 0 \text { as } \tau \rightarrow 0 .
$$

The mean-value theorem therefore enables us to write

$$
F\left(u_{k}^{\tau}+\tau\left\langle f_{k}^{\tau}\right\rangle\right) \leq F\left(u_{k}^{\tau}\right)+C \tau\left|\left\langle f_{k}^{\tau}\right\rangle\right|,
$$


which, together with (3.8), yields

$$
\begin{array}{r}
W_{1}\left(u_{k+1}^{\tau}-\left(u_{k}^{\tau}+\tau f_{k}^{\tau}\right)\right) \leq \int_{k \tau}^{(k+1) \tau} W_{1}(f(t, .)-\langle f(t, .)\rangle) \mathrm{d} t \\
+\int_{\Omega} F\left(u_{k}^{\tau}\right)-\int_{\Omega} F\left(u_{k+1}^{\tau}\right)+C \tau\left|\left\langle f_{k}^{\tau}\right\rangle\right| .
\end{array}
$$

Now, the right-hand side of (3.10) contains a telescopic sum and terms on which we have $L^{1}$ bounds thanks to (2.8). Hence, since $F \geq 0$ and $u_{0} \in L^{m}$ we get

$$
\sum_{k=0}^{\left[\tau^{-1} T\right]} W_{1}\left(u_{k+1}^{\tau}-u_{k}^{\tau}-\tau f_{k}^{\tau}\right) \leq C .
$$

Next we observe that

$$
\begin{aligned}
\left\|\partial_{t} u^{\tau}\right\|_{L^{1}\left((0, T),\left(\operatorname{Lip}^{\prime}, \widetilde{W}_{1}\right)\right)} & =\sum_{k=0}^{\left[\tau^{-1} T\right]} \widetilde{W}_{1}\left(u_{k+1}^{\tau}-u_{k}^{\tau}\right) \\
& \leq \int_{0}^{T}|\langle f(t, .)\rangle| \mathrm{d} t+\sum_{k=0}^{\left[\tau^{-1} T\right]} W_{1}\left(u_{k+1}^{\tau}-u_{k}^{\tau}-\tau\left\langle f_{k}^{\tau}\right\rangle\right) \\
& \leq C+\sum_{k=0}^{\left[\tau^{-1} T\right]}\left(W_{1}\left(u_{k+1}^{\tau}-u_{k}^{\tau}-\tau f_{k}^{\tau}\right)+\tau W_{1}\left(f_{k}^{\tau}-\left\langle f_{k}^{\tau}\right\rangle\right)\right.
\end{aligned}
$$

together with (3.11) and (2.8) we deduce

$$
\left\|\partial_{t} u^{\tau}\right\|_{L^{1}\left((0, T),\left(\operatorname{Lip}^{\prime}, \widetilde{W}_{1}\right)\right)} \leq C .
$$

The Euler-Lagrange equation of (2.5) from Lemma 3.1 reads

$$
-F^{\prime}\left(u_{k+1}^{\tau}\right) \in \mathrm{K}\left(u_{k+1}^{\tau}-u_{k}^{\tau}-\tau f_{k}^{\tau}\right)=\mathrm{K}\left(\frac{u_{k+1}^{\tau}-u_{k}^{\tau}}{\tau}-f_{k}^{\tau}\right)
$$

Note that by the very construction of the interpolations $u^{\tau}$ and $\widetilde{u}^{\tau},(3.13)$ can be rewritten as for a.e. $t \in[0, T]$

$$
-F^{\prime}\left(\widetilde{u}^{\tau}(t)\right) \in \mathrm{K}\left(\partial_{t} u^{\tau}(t)-f^{\tau}(t)\right) .
$$

i.e. $F^{\prime}\left(\widetilde{u}^{\tau}\right) \in \operatorname{Lip}_{1}$ and for every $\xi \in \operatorname{Lip}_{1}$ and for a.e. time one has

$$
\left\langle\partial_{t} u^{\tau}, F^{\prime}\left(\widetilde{u}^{\tau}\right)-\xi\right\rangle \leq\left\langle f^{\tau}, F^{\prime}\left(\widetilde{u}^{\tau}\right)-\xi\right\rangle .
$$


As already observed, given $k>0$ and $\theta \in \operatorname{Lip}_{1}, \xi:=F^{\prime}\left(\widetilde{u}^{\tau}\right)-T_{k}\left(F^{\prime}\left(\widetilde{u}^{\tau}\right)-\theta\right)$ belongs to $\operatorname{Lip}_{1}$, hence we have

$$
\left\langle\partial_{t} u^{\tau}, T_{k}\left(F^{\prime}\left(\widetilde{u}^{\tau}\right)-\theta\right)\right\rangle \leq\left\langle f^{\tau}, T_{k}\left(F^{\prime}\left(\widetilde{u}^{\tau}\right)-\theta\right)\right\rangle .
$$

Now we observe that if $t \in(k \tau,(k+1) \tau)$ by the strict convexity of $F$ and the fact that $T_{k}$ is nondecreasing, $T_{k}\left(F^{\prime}\left(\widetilde{u}^{\tau}(t)\right)-\theta\right)-T_{k}\left(F^{\prime}\left(u^{\tau}(t)\right)-\theta\right)$ has the sign of $\left(\widetilde{u}^{\tau}(t)-u^{\tau}(t)\right)$ i.e. that of $\partial_{t} u^{\tau}(t)$, hence

$$
\begin{aligned}
\frac{d}{d t} \int_{\Omega} \int_{0}^{u^{\tau}} T_{k}\left(F^{\prime}(s)-\theta\right) d s & =\left\langle\partial_{t} u^{\tau}, T_{k}\left(F^{\prime}\left(u^{\tau}\right)-\theta\right)\right\rangle \\
& \leq\left\langle\partial_{t} u^{\tau}, T_{k}\left(F^{\prime}\left(\widetilde{u}^{\tau}\right)-\theta\right)\right\rangle .
\end{aligned}
$$

With (3.16), this yields

$$
\frac{d}{d t} \int_{\Omega} \int_{0}^{u^{\tau}} T_{k}\left(F^{\prime}(s)-\theta\right) d s \leq\left\langle f^{\tau}, T_{k}\left(F^{\prime}\left(\widetilde{u}^{\tau}\right)-\theta\right)\right\rangle .
$$

Our aim now is of course to pass to the limit $\tau \rightarrow 0$ in (3.17). We first have:

Proposition 3.4. There exist a vanishing sequence of time steps $\tau_{n} \rightarrow 0$ as $n \rightarrow \infty$ and $u \in L^{\infty}\left((0, T), C^{0}(\bar{\Omega})\right)$, such that setting $u_{n}:=u^{\tau_{n}}$ and $\widetilde{u}_{n}:=\widetilde{u}^{\tau_{n}}$ one has:

$$
\begin{gathered}
u_{n} \rightarrow u \text { in } L^{p}\left((0, T), C^{0}(\bar{\Omega})\right), \forall p \in[1,+\infty) \\
\widetilde{u}^{n} \rightarrow u \text { in } L^{1}\left((0, T), C^{0}(\bar{\Omega})\right) \\
F^{\prime}\left(\widetilde{u}_{n}\right) \rightarrow F^{\prime}(u) \text { in } L^{p}\left((0, T), C^{0, \alpha}(\bar{\Omega})\right), \forall p \in[1,+\infty), \forall \alpha \in[0,1) \\
F^{\prime}(u(t, .)) \in \operatorname{Lip}_{1} \text { for a.e. } t
\end{gathered}
$$

Proof. Thanks to (3.7), $u^{\tau}$ is bounded in $L^{1}\left((0, T), C^{0, \beta}\right)$ and $\partial_{t} u^{\tau}$ is bounded in $L^{1}\left((0, T), \operatorname{Lip}^{\prime}\right)$, since the embedding $C^{0, \beta}(\Omega) \hookrightarrow C^{0}(\bar{\Omega})$ is compact and the embedding $C^{0}(\bar{\Omega}) \hookrightarrow \operatorname{Lip}(\Omega)^{\prime}$ is continuous (it is actually compact as well...), it follows from the Aubin-Lions-Simon Theorem (see [3], [16]) that $\left\{u^{\tau}\right\}_{\tau}$ has a cluster point $u$ in $L^{1}\left((0, T), C^{0}(\bar{\Omega})\right.$ ). For a suitable vanishing sequence of stepsizes we may thus assume that the corresponding sequence $u_{n}$ converges to $u$ in $L^{1}\left((0, T), C^{0}(\bar{\Omega})\right)$ but also (up to a further extraction) that $u_{n}(t,$.$) con-$ verges to $u(t,$.$) in C^{0}(\bar{\Omega})$ for a.e. $t$. Thanks to the uniform bound (3.7) with Lebesgue's dominated convergence theorem, we deduce (3.18).

As for $\widetilde{u}^{\tau}$, in addition to (3.7) and (3.12), we observe that

$$
\begin{array}{r}
\left\|\widetilde{u}^{\tau}-u^{\tau}\right\|_{L^{1}\left((0, T),\left(\operatorname{Lip}^{\prime}, \widetilde{W}_{1}\right)\right)}=\sum_{k=0}^{\left[\tau^{-1} T\right]} \int_{k \tau}^{(k+1) \tau} \widetilde{W}_{1}\left(\widetilde{u}^{\tau}(t)-u^{\tau}(t)\right) \mathrm{d} t \\
=\frac{\tau}{2} \sum_{k=0}^{\left[\tau^{-1} T\right]} \widetilde{W}_{1}\left(u_{k+1}^{\tau}-u_{k}^{\tau}\right) \leq C \tau
\end{array}
$$


where the last rightmost inequality follows from (3.12). Since $u_{n}$ obviously converges to $u$ in $L^{1}\left((0, T)\right.$, Lip $\left.^{\prime}\right)$, we deduce from the latter inequality that $\widetilde{u}^{n}$ is relatively compact in $L^{1}\left((0, T), \operatorname{Lip}^{\prime}\right)$, together with (3.7), the fact that the embedding $C^{0, \beta} \hookrightarrow C^{0}$ is compact, that the embedding $C^{0} \hookrightarrow \mathrm{Lip}^{\prime}$ is continuous and Lemma 9 in Simon [16], we can conclude that up to further extractions, $\widetilde{u}_{n}$ converges to $u$ in $L^{1}\left((0, T), C^{0}(\bar{\Omega})\right)$. Again, we may also assume as well that $\widetilde{u}_{n}(t,$.$) converges to u(t,$.$) in C^{0}(\bar{\Omega})$ for a.e. $t$. This implies that $F^{\prime}\left(\widetilde{u}_{n}(t,).\right)$ converges to $F^{\prime}(u(t,)$.$) in C^{0}(\bar{\Omega})$ for a.e. $t$ which in particular implies (3.21). Thanks to (3.7), for every $\alpha \in[0,1), F^{\prime}\left(\widetilde{u}_{n}(t,).\right)$ is relatively compact in $C^{0, \alpha}$ and thus converges to $F^{\prime}(u(t,)$.$) in C^{0, \alpha}$ for a.e. $t$, the $L^{p}\left((0, T), C^{0, \alpha}(\bar{\Omega})\right)$. Convergence in (3.20) thus simply follows again from the uniform bound (3.7) and Lebesgue's dominated convergence theorem.

\subsection{Proof of theorem 2.3}

We are now ready to prove our main result which in particular implies existence of weak solution of (1.1)-(1.2) via convergence of the JKO scheme (2.5), namely

Proposition 3.5. The limit function u from Proposition 3.4 is a weak solution of (1.1)(1.2).

Proof. Let $\theta \in \operatorname{Lip}_{1}$ and $\phi \in C_{c}^{1}\left([0, T), \mathbb{R}_{+}\right)$, multiplying (3.17) by $\phi$ and integrating by parts in time (observing that $\int_{\Omega} \int_{0}^{u^{\tau}} T_{k}\left(F^{\prime}(s)-\theta\right) d s$ is absolutely continuous) we first have:

$$
\begin{array}{r}
-\int_{0}^{T} \int_{\Omega} \dot{\phi}(t) \int_{0}^{u^{\tau}} T_{k}\left(F^{\prime}(s)-\theta\right) \mathrm{d} s \leq \phi(0) \int_{0}^{u_{0}} T_{k}\left(F^{\prime}(s)-\theta\right) \mathrm{d} s \\
+\int_{0}^{T} \phi(t)\left\langle f^{\tau}(t, .), T_{k}\left(F^{\prime}\left(\widetilde{u}^{\tau}(t, .)\right)-\theta\right)\right\rangle \mathrm{d} t .
\end{array}
$$

For the last term in this inequality, we remark that it can be rewritten as

$$
\begin{array}{r}
\int_{0}^{T} \phi(t)\left\langle f^{\tau}(t, .), T_{k}\left(F^{\prime}\left(\widetilde{u}^{\tau}(t, .)\right)-\theta\right)\right\rangle \mathrm{d} t=\int_{0}^{T}\left\langle f, \phi^{\tau} T_{k}\left(F^{\prime}\left(\widetilde{u}^{\tau}\right)-\theta\right)\right\rangle \\
=\int_{0}^{T} \phi^{\tau}(t)\left\langle f(t, .), T_{k}\left(F^{\prime}\left(\widetilde{u}^{\tau}(t, .)\right)-\theta\right)\right\rangle \mathrm{d} t
\end{array}
$$

with

$$
\phi^{\tau}(t):=\frac{1}{\tau} \int_{l \tau}^{(l+1) \tau} \phi(s) \mathrm{d} s, \text { for } t \in[l \tau,(l+1) \tau)
$$


It follows from proposition 3.4 that

$$
T_{k}\left(F^{\prime}\left(\widetilde{u}_{n}\right)-\theta\right) \rightarrow T_{k}\left(F^{\prime}(u)-\theta\right) \text { in } C^{0, \alpha_{0}}(\bar{\Omega}) \text { for a.e. } t \text {. }
$$

Remarking then that $\left\|\phi^{\tau}-\phi\right\|_{L^{\infty}} \rightarrow 0$ as $\tau \rightarrow 0$, thanks to (2.7), and Lebesgue's dominated convergence theorem, we get

$$
\lim _{n} \int_{0}^{T} \phi(t)\left\langle f^{\tau_{n}}(t, .), F^{\prime}\left(\widetilde{u}_{n}(t, .)-\theta\right\rangle \mathrm{d} t=\lim _{n} \int_{0}^{T} \phi(t)\left\langle f(t, .), F^{\prime}(u(t, .))-\theta\right\rangle \mathrm{d} t .\right.
$$

Taking $\tau=\tau_{n}$, letting $n \rightarrow \infty$ and using proposition 3.4, we thus easily deduce that $u$ is a weak solution of (1.1)-(1.2).

\section{$4 \quad L^{1}$-contraction, uniqueness and stability}

Theorem 4.1. Assume that $f \in L^{1}\left((0, T), L^{1}(\Omega)\right)$. Let $u_{1}$ and $u_{2}$ be two weak solutions of (1.1)-(1.2), then

$$
\frac{d}{d t} \int_{\Omega}\left|u_{1}(t, x)-u_{2}(t, x)\right| d x \leq 0 \quad \text { in } \mathcal{D}^{\prime}(0, T)
$$

In particular, for any $u_{0} \in L^{1}$ and $f \in L^{1}\left((0, T), L^{1}(\Omega)\right)$, problem (1.1)-(1.2) has at most one weak solution such that $u(0)=u_{0}$.

Proof. Let $u_{1}$ and $u_{2}$ be two weak solutions. Dividing (2.14) by $k$ and letting $k$ tend to $0^{+}$, for $i=1,2$, thanks to the fact that $f \in L^{1}\left((0, T), L^{1}(\Omega)\right)$ and Lebesgue's dominated convergence theorem, we have ${ }^{3}$, for any $\theta \in \operatorname{Lip}_{1}$

$$
\frac{d}{d t} \int_{\Omega} \int_{0}^{u_{i}(t, x)} \operatorname{sign}\left(F^{\prime}(r)-\theta(x)\right) d r \mathrm{~d} x \leq\left\langle f, \operatorname{sign}\left(F^{\prime}\left(u_{i}(t)\right)-\theta\right)\right\rangle, \quad \text { in } \mathcal{D}^{\prime}(0, T) .
$$

In particular, if $\theta$ is of the form $\theta=F^{\prime}(\eta)$, we get

$$
\begin{array}{r}
\frac{d}{d t} \int_{\Omega} \int_{0}^{u_{i}(t, x)} \operatorname{sign}(r-\eta(x)) d r \mathrm{~d} x=\frac{d}{d t} \int_{\Omega} \int_{\eta(x)}^{u_{i}(t, x)} \operatorname{sign}(r-\eta(x)) d r \mathrm{~d} x \\
\leq\left\langle f, \operatorname{sign}\left(F^{\prime}\left(u_{i}(t)\right)-F^{\prime}(\eta)\right)\right\rangle, \quad \text { in } \mathcal{D}^{\prime}(0, T) .
\end{array}
$$

Thus

$$
\frac{d}{d t} \int_{\Omega}\left|u_{i}(t, x)-\eta(x)\right| \mathrm{d} x \leq\left\langle f(t), \operatorname{sign}\left(u_{i}(t)-\eta\right)\right\rangle, \quad \text { in } \mathcal{D}^{\prime}(0, T) .
$$

\footnotetext{
${ }^{3}$ Here and in the sequel, sign refers to the selection of the sign function which takes value 0 at 0 .
} 
Now, we use doubling and dedoubling variables techniques to prove the $L^{1}$ contraction principle. Let $\sigma \in \mathcal{D}\left((0, T)^{2}\right), \sigma \geq 0$, if we take for $i=1, \eta(x)=F^{\prime}\left(u_{2}(s, x)\right)$, (s fixed for the moment) in the inequality above, we get

$$
\begin{aligned}
& -\int_{0}^{T} \int_{\Omega} \partial_{t} \sigma(t, s)\left|u_{1}(t, x)-u_{2}(s, x)\right| \mathrm{d} x \mathrm{~d} t \\
& \leq \int_{0}^{T} \int_{\Omega} f(t, x) \operatorname{sign}\left(u_{1}(t, x)-u_{2}(s, x)\right) \sigma(t, s) \mathrm{d} x \mathrm{~d} t
\end{aligned}
$$

In a similar way, using $\eta(x)=F^{\prime}\left(u_{1}(t, x)\right)$ (with $t$ fixed) for $u_{2}$, we have

$$
\begin{aligned}
& -\int_{0}^{T} \int_{\Omega} \partial_{s} \sigma(t, s)\left|u_{1}(t, x)-u_{2}(s, x)\right| \mathrm{d} x d s \\
& \leq \int_{0}^{T} \int_{\Omega} f(s, x) \operatorname{sign}\left(u_{1}(t, x)-u_{2}(s, x)\right) \sigma(t, s) \mathrm{d} x d s
\end{aligned}
$$

Integrating the preceding equations with respect to $s$ and $t$, respectively, and adding the resulting inequalities, we thus get

$$
\begin{aligned}
& -\int_{0}^{T} \int_{0}^{T} \int_{\Omega}\left|u_{1}(t, x)-u_{2}(s, x)\right|\left(\partial_{t} \sigma(t, s)+\partial_{s} \sigma(t, s)\right) \mathrm{d} x \mathrm{~d} t d s \\
& \leq \int_{0}^{T} \int_{0}^{T} \int_{\Omega}|f(t, x)-f(s, x)| \sigma(t, s) \mathrm{d} x \mathrm{~d} t d s .
\end{aligned}
$$

Now, to dedouble variables, given a small $\varepsilon>0$ we take $\sigma=\sigma_{\varepsilon}$ of the form

$$
\sigma_{\varepsilon}(t, s)=\frac{\phi(t)}{\varepsilon} \rho\left(\frac{s-t}{\varepsilon}\right)
$$

with $\phi \in \mathcal{D}(0, T), \phi \geq 0$ and $\rho \geq 0, \rho \in \mathcal{D}(-1,1)$, even, and such that $\int_{\mathbb{R}} \rho=1$. We then have

$$
\partial_{t} \sigma_{\varepsilon}(t, s)+\partial_{s} \sigma_{\varepsilon}(t, s)=\frac{\dot{\phi}(t)}{\varepsilon} \rho\left(\frac{t-s}{\varepsilon}\right) .
$$

Performing the change of variables $(t, s) \mapsto\left(t, \frac{s-t}{\varepsilon}\right)$, we thus arrive at

$$
-I_{\varepsilon} \leq J_{\varepsilon}
$$

where

$$
I_{\varepsilon}:=\int_{-1}^{1} \int_{0}^{T} \int_{\Omega}\left|u_{1}(t, x)-u_{2}(t+\varepsilon \tau, x)\right| \rho(\tau) \dot{\phi}(t) \mathrm{d} x \mathrm{~d} t \mathrm{~d} \tau
$$


and

$$
J_{\varepsilon}:=\int_{-1}^{1} \int_{0}^{T} \int_{\Omega}|f(t, x)-f(t+\varepsilon \tau, x)| \rho(\tau) \phi(t) \mathrm{d} x \mathrm{~d} t \mathrm{~d} \tau .
$$

Now using the fact that if $g \in L^{1}((0, T) \times \Omega)$, then for $a$ and $b$ such that $0<a<b<T$, $\|g(.+h, .)-g(., .)\|_{L^{1}((a, b) \times \Omega)}$ tends to 0 uniformly as $|h| \rightarrow 0$ (see for instance Chapter 4 of [4] for details), we easily obtain

$$
\lim _{\varepsilon \rightarrow 0^{+}} I_{\varepsilon}=\int_{0}^{T} \int_{\Omega}\left|u_{1}(t, x)-u_{2}(t, x)\right| \dot{\phi}(t) \mathrm{d} x \mathrm{~d} t
$$

and, since $f \in L^{1}((0, T) \times \Omega)$, we similarly have

$$
\lim _{\varepsilon \rightarrow 0^{+}} J_{\varepsilon}=0
$$

Letting $\varepsilon$ tend to $0^{+}$in (4.3), we deduce the desired contraction result (4.1).

Remark 4.2. Exactly the same proof as above, gives the following stability result for weak solutions $u_{1}$ and $u_{2}$ associated to different $\left(L^{1}((0, T) \times \Omega)\right)$ sources, respectively $f_{1}$ and $f_{2}$ :

$$
\frac{d}{d t}\left\|u_{1}(t, .)-u_{2}(t, .)\right\|_{L^{1}(\Omega)} \leq\left\|f_{1}(t, .)-f_{2}(t, .)\right\|_{L^{1}(\Omega)}
$$

and in particular

$$
\left\|u_{1}(t, .)-u_{2}(t, .)\right\|_{L^{1}(\Omega)} \leq\left\|u_{1}(0, .)-u_{2}(0, .)\right\|_{L^{1}(\Omega)}+\int_{0}^{t}\left\|f_{1}(s, .)-f_{2}(s, .)\right\|_{L^{1}(\Omega)} \mathrm{d} s .
$$

\section{$5 \quad$ Variants and concluding remarks}

We have proposed an elementary Euler scheme à la JKO with $W_{1}$ to deal with nonlinear evolution equations of the form (1.1)-(1.2) and addressed stability and uniqueness issues thanks to an $L^{1}$-contraction argument. We presented the $L^{1}$-contraction directly at the level of the PDE, but another approach, leading to the same conclusion, would have been to consider contraction at the discretized in time level (in a similar way as the estimate of Proposition 3.2) and conclude by the classical semi-group theory in Banach spaces of Crandall and Liggett [8]. Indeed, thanks to Proposition 3.2, it is possible to handle the evolution problem (1.1)-(1.2) by using the classical semi-group theory in the Banach space $L^{1}(\Omega)$, whenever the source term is $L^{1}$ in space. In particular, one sees that (4.2) is closely connected to the notion of integral solution in the sense of non linear semi-group theory in $L^{1}(\Omega)$. If the source term is regular enough, we believe that it is possible to prove the existence of a weak solution in the standard sense (cf. remark 2.2). Thanks to 
remark 2.2, this solution coincides with ours and the uniqueness holds true if the source term remains in $L^{1}$.

Let us stress the fact that the $W_{1}$-JKO scheme is constructive. We indeed believe that since the scheme consists in a sequence of relatively simple convex minimization problems, it is well suited for numerical purposes but we leave this aspect for future research.

An easy extension of the $W_{1}-\mathrm{JKO}$ approach concerns the case of a reaction term in the right-hand side i.e.

$$
\partial_{t} u-\operatorname{div}\left(a \nabla F^{\prime}(u)\right)=f(u),(t, x) \in(0, T) \times \Omega,\left.u\right|_{t=0}=u_{0},
$$

again constrained by (1.2). If the reaction term $f(u)$ is sublinear, i.e. there exists a positive constant $C$ such that

$$
|f(u)| \leq C(1+|u|), \forall u \in \mathbb{R}
$$

and if we modify (2.5) in a straighforward way by

$$
u_{k+1}^{\tau} \in \operatorname{argmin}_{u}\left\{W_{1}\left(u-\left(u_{k}^{\tau}+\tau f\left(u_{k}^{\tau}\right)\right)\right)+\int_{\Omega} F(u) \mathrm{d} x:\left\langle u-u_{k}^{\tau}-\tau f\left(u_{k}^{\tau}\right)\right\rangle=0\right\}
$$

then, thanks to (5.2), one can obtain similar estimates as in section 3 to deduce convergence of the scheme (5.3) as $\tau \rightarrow 0^{+}$to a solution of (5.1)-(1.2). If, in addition $f$ is Lipschitz, then it follows directly from (4.5) and Gronwall's Lemma that we also have uniqueness and stability in $L^{1}$. To make things simple we have considered a power convex nonlinearity for $F$, but this is not really essential, what is important is that $F^{\prime}$ is an homeorphism. An interesting limit case, out of the scope of the present analysis, is when $F^{\prime}$ is a general monotone graph, possibly set-valued or empty-valued such as in the compression molding model of Aronsson and Evans [1].

We have also have left unanswered two questions that seem natural to us. The first one is what happens if the source term $f$ is only $L^{1}\left((0, T), \operatorname{Lip}^{\prime}\right)$ : can one expect convergence of the JKO scheme, and more generally, does there exist a weak solution to (1.1)-(1.2) in this case? The second one is the uniqueness of weak solutions to the Cauchy problem when the source is not $L^{1}((0, T) \times \Omega)$ but only $\left.L^{1}\left((0, T) \text {, } \operatorname{Lip}^{\prime}\right)^{\prime}\right)$ or $L^{1}\left((0, T),\left(C^{0, \alpha_{0}}(\bar{\Omega})\right)^{\prime}\right)$, we actually suspect that uniqueness is false in such irregular cases but have not found any counterexample.

Acknowledgements: G.C. and N.I gratefully acknowledge the hospitality of the Mathematics and Statistics Department at UVIC (Victoria, Canada) where part of the present research was done. G.C. benefited from support from the ANR, through the projects ISOTACE (ANR-12- MONU-0013), MAGA ( ANR-16-CE40-0014) and from INRIA through the équipe-projet commune MOKAPLAN. 


\section{References}

[1] G. Aronsson and L. C. Evans. An asymptotic model for compression molding. Indiana Univ. Math. J., 51(1):1-36, 2002.

[2] G. Aronsson, L. C. Evans, and Y. Wu. Fast/slow diffusion and growing sandpiles. J. Differential Equations, 131(2):304-335, 1996.

[3] Jean-Pierre Aubin. Un théorème de compacité. C. R. Acad. Sci. Paris, 256:50425044, 1963.

[4] Haïm Brezis. Analyse fonctionnelle. Collection Mathématiques Appliquées pour la Maîtrise. [Collection of Applied Mathematics for the Master's Degree]. Masson, Paris, 1983. Théorie et applications. [Theory and applications].

[5] Piermarco Cannarsa and Pierre Cardaliaguet. Representation of equilibrium solutions to the table problem for growing sandpiles. J. Eur. Math. Soc. (JEMS), 6(4):435-464, 2004 .

[6] Piermarco Cannarsa, Pierre Cardaliaguet, and Carlo Sinestrari. On a differential model for growing sandpiles with non-regular sources. Comm. Partial Differential Equations, 34(7-9):656-675, 2009.

[7] J. Carillo. Entropy solutions for nonlinear degenerate problems. Arch. Rational Mech. Anal., 147(4):269-361, 1999.

[8] M. G. Crandall and T. M. Liggett. Generation of semi-groups of nonlinear transformations on general Banach spaces. Amer. J. Math., 93:265-298, 1971.

[9] Luigi De Pascale and Chloé Jimenez. Duality theory and optimal transport for sand piles growing in a silos. Adv. Differential Equations, 20(9-10):859-886, 2015.

[10] S. Dumont and N. Igbida. On a dual formulation for the growing sandpile problem. European J. Appl. Math., 20(2):169-185, 2009.

[11] L. C. Evans, M. Feldman, and R. F. Gariepy. Fast/slow diffusion and collapsing sandpiles. J. Differential Equations, 137(1):166-209, 1997.

[12] N. Igbida. Evolution monge-kantorovich equation. J. Differential Equations, 225(7):1383-1407, 2013.

[13] Richard Jordan, David Kinderlehrer, and Felix Otto. The variational formulation of the Fokker-Planck equation. SIAM J. Math. Anal., 29(1):1-17, 1998. 
[14] David Kinderlehrer and Noel J. Walkington. Approximation of parabolic equations using the Wasserstein metric. Mathematical Modelling and Numerical Analysis, 33(4):837-852, 1999.

[15] Leonid Prigozhin. Variational model of sandpile growth. European J. Appl. Math., 7:225-235, 1996.

[16] Jacques Simon. Compact sets in the space $L^{p}(0, T ; B)$. Ann. Mat. Pura Appl. (4), 146:65-96, 1987.

[17] C. Villani. Topics in Optimal Transportation, volume 58 of Graduate Studies in Mathematics. American Mathematical Society, Providence, 2003. 